\title{
Treatment of immune recovery vitritis with local steroids
}

Hugo W A Henderson, Suzanne M Mitchell

\begin{abstract}
Aims-To report a series of patients requiring treatment for falling visual acuity associated with immune recovery vitritis, a recently described syndrome of a predominantly vitreous inflammatory reaction in patients with AIDS and cytomegalovirus (CMV) retinitis.
\end{abstract}

Methods-The medical records of all patients requiring treatment for falling visual acuity associated with immune recovery vitritis were reviewed between March 1996 and March 1998.

Results-Nine eyes in seven patients required treatment for falling visual acuity. All patients had inactive CMV retinitis and had received highly active antiretroviral treatment including a protease inhibitor. Vitreous inflammation developed at a mean of 5.5 months (range 1-14) after starting a protease inhibitor. The onset of inflammation correlated with a mean rise in $\mathrm{CD4}^{+}$lymphocyte levels of $83 \times 10^{6} / 1$ (range 30-128). The visual acuity fell by a mean of 2.8 Snellen lines (range 1-4) before treatment, and rose by a mean of 1.9 Snellen lines (range 0-4) after treatment with orbital floor steroids. The mean time interval between treatment with orbital floor steroids and improvement in visual acuity was 3.5 weeks (range 1-8). Following treatment the visual acuity improved or remained stable in all nine eyes, eight eyes returning to within one line of their preinflammation Snellen visual acuity. No eyes developed reactivation or progression of CMV retinitis after treatment, and none developed any other pathology.

Conclusions-Orbital floor steroids appear to be have a useful role in the treatment of persistent immune recovery vitritis where the visual acuity is compromised.

(Br f Ophthalmol 1999;83:540-545)

St Stephen's Centre, Chelsea and

Westminster Hospital,

London

H W A Henderson

S M Mitchell

Correspondence to: Suzanne M Mitchell, St Stephen's Centre, Chelsea and Westminster Hospital, Fulham Road, London SW10 9NH.

Accepted for publication 25 November 1998
Immune recovery vitritis (IRV) is a recently described syndrome of a predominantly vitreous inflammatory reaction in patients with acquired immune deficiency syndrome (AIDS) and cytomegalovirus (CMV) retinitis. The syndrome is associated with increased immunocompetence as a result of highly active antiretroviral treatment (HAART) including protease inhibitors. ${ }^{12}$ Cystoid macular oedema (CMO) and epiretinal membranes have been documented in association with the syndrome. ${ }^{1}$
AIDS related CMV retinitis generally occurs after the $\mathrm{CD} 4^{+}$lymphocyte level has fallen to less than $50 \times 10^{6}$ cells $/ 1$ and rarely occurs at $\mathrm{CD} 4^{+}$lymphocyte levels of greater than $100 \times$ $10^{6}$ cells $/ 1 .^{34}$ This profound immunodeficiency may have a protective effect against complications induced by inflammation, although HIV related vitritis, ${ }^{5}$ and $\mathrm{CMO}$ with AIDS related CMV retinitis have been reported in patients who are not taking protease inhibitors. ${ }^{56}$ AIDS related CMV retinitis was typically associated with little or no vitreous inflammatory reaction even in the presence of extensive disease in the pre-HAART era. ${ }^{7}$ With the advent of HAART many patients have experienced an improvement in their immune status associated with rising $\mathrm{CD}^{+}$lymphocyte levels and decreasing HIV RNA levels. ${ }^{8}{ }^{9}$ IRV is associated with a rise in $\mathrm{CD}^{+}$lymphocyte levels in most patients. ${ }^{210}$

The clinical picture of IRV is still evolving. Karravellas et al report that immune recovery vitritis in their group of patients was persistent, while Zegans et al reported a transient vitritis with improvement in visual acuity within 6 weeks of initial diagnosis regardless of treatment. ${ }^{12}$

Vitritis in patients with HIV infection and AIDS can result from retinal or choroidal infection with multiple opportunistic pathogens including Mycobacterium species, Treponema pallidum, Toxoplasma gondii, fungi, as well as members of the herpesvirus family. ${ }^{71-15}$ Dense vitritis has recently been reported as the primary manifestation of ocular syphilis in patients with AIDS in the absence of other posterior segment findings or of substantial anterior segment inflammation. ${ }^{14}$ The differential diagnosis between these conditions and IRV may not be straightforward especially when faced with changing patterns of ocular disease in patients with HIV infection. Previously, oral steroids have been reported to be a risk factor for CMV retinitis in HIV positive patients with a low $\mathrm{CD} 4^{+}$lymphocyte count ${ }^{15}$; however, successful steroid treatment for IRV has been reported without complications. ${ }^{12}$ It is important to exclude these possibilities before embarking on steroid therapy for those patients in whom the IRV was associated with a fall in visual acuity sufficient to necessitate treatment.

We evaluate the results of treatment with local steroids in nine eyes of seven patients with IRV.

\section{Methods}

The case notes of all patients in the department of HIV and ophthalmology at the Chelsea and Westminster Hospital, London, 


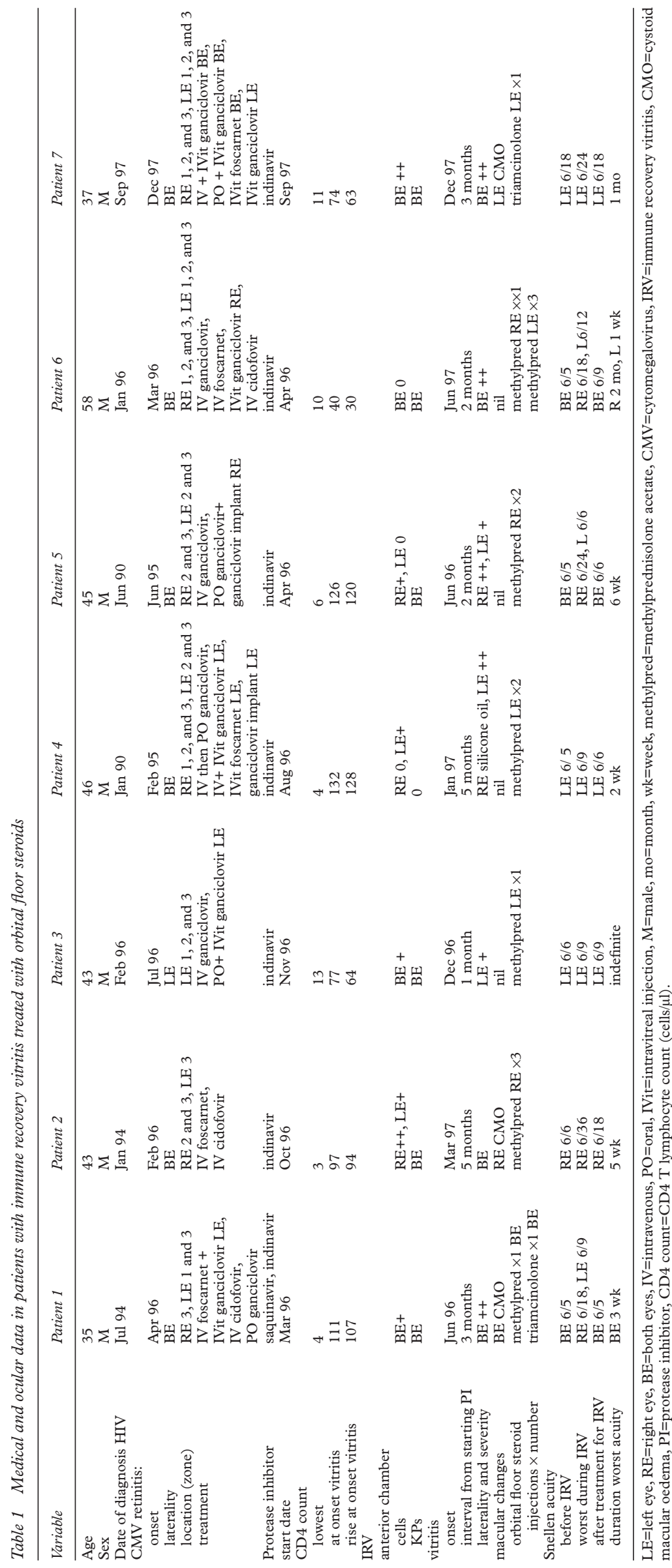

who had been treated with orbital floor steroids between March 1996 and March 1998 were studied. Nine eyes were identified of seven patients who had been treated with orbital floor steroids for IRV. All patients were being treated with antiretroviral combination therapy including protease inhibitors, had inactive CMV retinitis, and rising $\mathrm{CD} 4^{+}$ lymphocyte levels. None of the patients was taking rifabutin, cidofovir, or fomivirsen (ISSIS-2922) before the onset of vitreous inflammation.

Indications for treatment were persistent reduction in vision or symptoms unacceptable to the patient resulting from vitritis or the complications of vitritis. All patients except patient 2 had been treated with topical dexamethasone four times daily for at least 3 weeks with no improvement before treatment with orbital floor steroids. None of the patients was treated with systemic steroids. Orbital floor injections of methylprednisolone acetate $40 \mathrm{mg}$ or triamcinolone $20 \mathrm{mg}$ (as indicated in Table 1) were given using a 25 gauge needle $5 / 8$ inch in length inserted through the skin and Tenon capsule at the junction of the medial two thirds and lateral one third of the lower eyelid.

\section{Results}

During the 2 years of this study a total of 80 patients with CMV retinitis who were treated with HAART were followed longitudinally. Most patients developed a mild transient vitritis not requiring treatment. Only nine eyes of seven patients developed IRV of such severity that therapeutic intervention was deemed necessary. Repeat injections were used in the treatment of four eyes (as indicated in Table 1). Four eyes of three patients developed clinical $\mathrm{CMO}$, confirmed by fluorescein angiography in three eyes. All eyes that developed clinical CMO were treated with orbital floor steroids. None of the other 73 patients treated with HAART developed CMO.

The development of IRV occurred at a mean of 5.5 months (range 1-14 months) after HAART was commenced, and correlated with a mean rise in $\mathrm{CD} 4^{+}$lymphocyte levels of $83 \times 10^{6} / 1$ (range $30-128$ ). The vitreous inflammatory reaction developed only in the eye with CMV retinitis. Before treatment the mean fall in visual acuity was 2.8 Snellen lines (range 1-4), and lasted for a mean of 3.5 weeks (range 1-8). Repeat injections were used in the treatment of four eyes (as indicated in Table 1). The mean increase in visual acuity after treatment with orbital floor steroids was 1.9 Snellen lines. Following treatment eight of the nine eyes returned to within one line of their preinflammation Snellen visual acuity. In one eye (of patient 2) the Snellen visual acuity improved by one line after treatment but never recovered to the level achieved before the onset of vitritis.

No eyes developed reactivation or progression of CMV retinitis after treatment, 

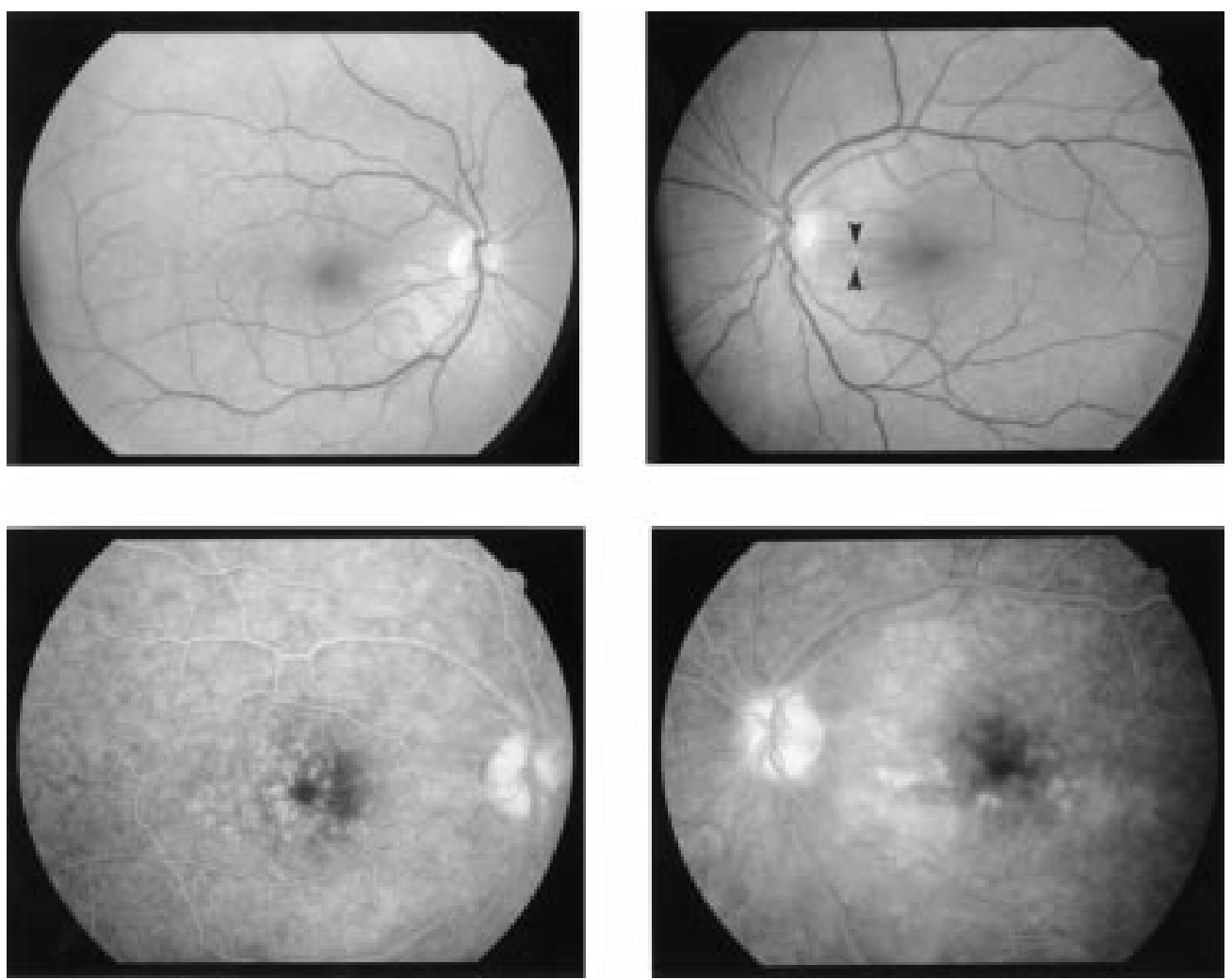

Figure 1 Case 1. Top: red-free photographs of right and left fundus showing inactive macular CMV retinitis in left eye. Bottom: late venous phase fluorescein angiograms of right and left eyes showing petaloid fluorescein leakage of CMO.

and none developed any other pathology. None of the patients developed a granulomatous uveitis.

A summary of the clinical histories and results is shown in Table 1 . Three of the cases of particular interest are reported in detail below.

CASE 1

A 35 year old man who was diagnosed HIV positive in July 1994. Combination antiretroviral therapy including the use of a protease inhibitor was started in March 1996 at a CD4 ${ }^{+}$ lymphocyte level of $4 \times 10^{6}$ cells $/ 1$. He developed bilateral CMV retinitis in April 1996 (zone 3 in the right eye and zone 1 in the left eye) when his $\mathrm{CD}^{+}$lymphocyte level remained unchanged. Induction therapy with intravenous foscarnet and two intravitreal injections of ganciclovir to the left eye was initially successful and maintenance therapy was continued with intravenous foscarnet. The visual acuity was maintained at $6 / 5$ in both eyes. This was accompanied by the development of mild vitreous activity $(+)$ in both eyes as the $\mathrm{CD} 4^{+}$lymphocyte level rose to $111 \times 10^{6}$ cells/ 16 weeks later. ${ }^{16}$ After 6 months the CMV retinitis became bilaterally active in zone 3 in both eyes at a CD4 $4^{+}$lymphocyte level of $116 \times$ $10^{6}$ cells $/ 1$, and intravenous cidofovir was commenced. The intraocular pressure was $16 \mathrm{~mm}$ $\mathrm{Hg}$ bilaterally. Within 1 month the patient developed asymptomatic mild anterior cell activity $(+)$ and moderate vitritis $(++)$ bilaterally. The visual acuity was $6 / 6$ in both eyes. Treatment was commenced with topical steroids and bilateral orbital floor injections of methylprednisolone acetate. One month later the visual acuity was right eye $6 / 18$, and left $6 / 9$. The intraocular pressures remained 16 $\mathrm{mm} \mathrm{Hg}$ in both eyes. The vitreous activity had improved to cells + bilaterally; however, fundal examination revealed CMO confirmed by fluorescein angiography (Fig 1). Treatment was commenced with oral acetazolamide. Within 2 weeks the visual acuity had improved to 6/9 bilaterally and clinically the CMO had improved. The intraocular pressures were 4 $\mathrm{mm} \mathrm{Hg}$ bilaterally.

After a further 2 weeks the patient discontinued acetazolamide as he was suffering malaise, and 2 days later the visual acuities had fallen to right eye $6 / 60$ and left $6 / 18$, the CMO had clinically recurred, and the intraocular pressures were $10 \mathrm{~mm} \mathrm{Hg}$ bilaterally. Bilateral orbital floor injections of triamcinolone and flurbiprofen (Froben) $100 \mathrm{mg}$ were given. No improvement in clinical signs was seen. Oral acetazolamide was restarted and the visual acuity had improved to $6 / 9$ bilaterally but the intraocular pressure fell to $6 \mathrm{~mm} \mathrm{Hg}$ bilaterally. One week later the patient again became intolerant of acetazolamide. The $\mathrm{CD} 4^{+}$lymphocyte level had risen to $150 \times 10^{6}$ cells $/ 1$ and cidofovir was stopped and oral ganciclovir maintenance was given. The acetazolamide dose was reduced, and was stopped 2 weeks later. The visual acuity remained $6 / 5$ bilaterally and there was no recurrence of CMO until his death 1 year later.

CASE 2

A 43 year old man who was diagnosed HIV positive in January 1994 developed zone 2 and 
3 CMV retinitis in the right eye in February 1996 which was successfully treated with induction and then maintenance intravenous foscarnet maintaining a vision of $6 / 6$ in both eyes. Eight months later combination antiretroviral therapy including the use of a protease inhibitor was started at a $\mathrm{CD}^{+}$lymphocyte level of $3 \times 10^{6}$ cells $/ 1$. Five months later his $\mathrm{CD}^{+}$lymphocyte level had risen to $97 \times 10^{6}$ cells/1 when he developed a moderate vitritis $(++)$ in his right eye with a visual acuity of $6 / 24$. The left eye remained quiet. An orbital floor injection of methylprednisolone acetate was given to the right orbit. The vision in the right eye improved over the following 2 weeks to $6 / 9$ and the vitritis resolved. One month later active CMV retinitis developed in zone 3 of the left eye, and maintenance foscarnet was changed to induction intravenous cidofovir. Two months later, at a $\mathrm{CD}^{+}$lymphocyte level of $100 \times 10^{6}$ cells/1, a moderate vitritis (++) recurred in both eyes complicated by clinical $\mathrm{CMO}$ in the right eye reducing the vision in that eye to $6 / 36$. A further right orbital floor injection of methylprednisolone acetate was given. Three weeks later, although the vitritis had improved $(+)$ in the right eye, there was no improvement in the CMO and oral acetazolamide $250 \mathrm{mg}$ four times daily was started. Two weeks later the visual acuity in the right eye had improved to $6 / 18$ and clinically the CMO had improved. He remained on oral acetazolamide for 2 months, and the vision has remained stable in the right eye with a persistent mild vitritis, which has required one further orbital floor injection of methylprednisolone acetate.

CASE 7

A 37 year old man who was diagnosed HIV positive in September 1997 after developing pulmonary tuberculosis at a $\mathrm{CD} 4^{+}$lymphocyte level of $11 \times 10^{6} / 1$. Antituberculous therapy and combination antiretroviral treatment including a protease inhibitor was started. He developed bilateral CMV retinitis in zones 2 and 3 of both eyes 3 months later at a $\mathrm{CD} 4^{+}$lymphocyte level of $74 \times 10^{6} / 1$. This was associated with a bilateral moderate anterior uveitis and vitritis $(++)$. Induction therapy with intravenous ganciclovir and bilateral injections of ganciclovir and then maintenance treatment with oral ganciclovir and fortnightly injections of ganciclovir to both eyes controlled the disease initially and he retained visual acuities of right eye $6 / 5$ and left $6 / 18$. After 1 month the CMV retinitis recurred in zones 1, 2, and 3 of both eyes. There was a continuing moderate bilateral anterior uveitis and vitritis $(++)$. Bilateral weekly intravitreal injections of foscarnet controlled the recurrence. Three weeks later the patient complained of a reduction in his left vision. Visual acuities were right $6 / 6$ and left $6 / 24$. There was a bilateral moderate anterior uveitis and vitritis $(++)$, and CMO in the left eye confirmed by fluorescein angiography. An orbital floor injection of triamcinolone $40 \mathrm{mg}$ was given to the left eye and topical steroids to both eyes. One week later there was no improvement and slow release oral acetazolamide $250 \mathrm{mg}$ twice daily was started. One week later there was still no improvement and an intravitreal injection of ganciclovir was given to the left eye. One week later the vitritis on the left was less intense, the CMO had improved clinically, and the visual acuity had improved to $6 / 18$.

\section{Discussion}

Following the introduction of protease inhibitors in 1996 and their use in combination with nucleoside analogues in HAART, the pattern of disease in AIDS related CMV retinitis has been changing. Rates of progression of CMV retinitis have decreased, long lasting remission of CMV retinitis is reported in patients without specific anti-CMV maintenance therapy, and there is evidence that the effect of protease inhibitors as part of combined antiretroviral therapy alone can result in regression of CMV retinitis. ${ }^{17-20}$ Patients with CMV retinitis who commence HAART have been shown to have a dramatically increased survival. ${ }^{21}$

Some of these patients have vision that has been severely compromised by previous episodes of CMV retinitis; however, the lack of progression of CMV retinitis has, for the present, improved the prognosis for retaining the remaining vision. In most patients IRV represents a transient phenomenon with no further threat to vision. However, in a few patients it appears to persist, and threaten the vision at a time when patients are starting to feel the benefits to their general health of an improved immune status. Data presented here suggest that orbital floor steroids have a useful role to play in the treatment of patients where visual acuity is compromised.

The results of treatment with orbital floor steroids are encouraging with eight out of the nine eyes returning to within one line of their preinflammation Snellen visual acuity, and no evidence of complications of treatment. None of the eyes treated with orbital floor steroids developed a recurrence of CMV retinitis, and none developed any other retinal lesion, or a vitreous abscess.

In contrast with AIDS related CMV retinitis, CMV retinitis in immunosuppressed patients following transplant surgery, however, is associated with vitreous inflammation, and this inflammation has been observed to increase following reduction of immunosuppressive drugs in an effort to control the infection. ${ }^{22}$ The onset of IRV in patients with CMV retinitis correlates with rising $\mathrm{CD}^{+}$lymphocyte counts following the initiation of HAART. Zegans et al postulate that the inflammatory findings represent an enhanced immune response to CMV in the retina.

Connors et al have examined changes in $\mathrm{CD}^{+}$lymphocyte surface marker phenotype and antigen receptor repertoire during the course of HIV infection and following therapy. They have reported that $\mathrm{CD}^{+}$lymphocyte death mediated by HIV-1 infection may result in a preferential decline in the number of naive $\mathrm{CD}^{+}$lymphocytes and disruptions of the $\mathrm{CD}^{+}$lymphocyte repertoire that are not immediately corrected by HAART. ${ }^{23}$ Thus, these patients retain holes in their immune defences even after their $\mathrm{CD} 4^{+}$lymphocyte 
counts have risen. In our series of patients, as in others, there was wide variation in duration of HAART, and in the rise in $\mathrm{CD}^{+}$lymphocyte levels, before onset of IRV. Also three patients developed active CMV retinitis at $\mathrm{CD} 4{ }^{+}$lymphocyte counts of $74 \times 10^{6} / 1$ or greater after starting HAART. These observations may reflect differences in the degree of disruption of the $\mathrm{CD} 4^{+}$lymphocyte repertoire capable of response to $\mathrm{CMV}$, or differences in the degree of depletion of surviving clones and the time needed to re-establish these $\mathrm{CD}^{+}$ lymphocyte pools.

There appears to be a spectrum of severity of IRV. Most patients with inactive AIDS related CMV retinitis and substantial improvements in $\mathrm{CD}^{+}$lymphocyte levels following HAART developed vitritis. This was generally mild and transient. The patients in this series represent a small but significant subpopulation with a more severe or persistent vitritis leading in some cases to CMO and in all cases to a fall in visual acuity.

Zegans et al suggest that vitreous inflammation only starts when immune reconstitution has developed sufficiently for a response to be mounted against the antigen and that it ends when inactivation of the virus halts or production of antigens is altered such that there is no longer a stimulus for inflammation. It may be that patients with persistent IRV have suboptimal reconstitution of the $\mathrm{CD} 4^{+}$lymphocyte repertoire capable of response to $\mathrm{CMV}$, or that continuous replication of virus or enhanced antigenicity of some strains may result in more severe or prolonged inflammation. It is also possible that other foci of CMV infection elsewhere in the body may increase the immune response in the eye during immune reconstitution.

Three of the five patients with vision loss of two lines or greater (patients 1, 2, and 7 described in detail earlier) developed CMO associated with the uveitis. The development of CMO did not appear to be associated with the zone of CMV retinitis. Treatment options for uveitic CMO include periocular and systemic corticosteroids and acetazolamide. ${ }^{24-26}$ In this series topical corticosteroids were used where there was clinically significant anterior segment inflammation. The main advantage of periocular corticosteroids is the production of good local drug levels avoiding the potential problems of systemic corticosteroids in this immunosuppressed population. However, treatment cannot be rapidly withdrawn if complications develop. Systemic acetazolamide needs to be used with care in patients with renal failure or taking medications known to have renal toxicity. In addition, patients on multiple medications often find the known side effects less tolerable. The CMO responded well to treatment with oral acetazolamide in patients 1 and 2 suggesting that oral acetazolamide may have an important role in the treatment of IRV when associated with CMO.

Cidofovir is known to be associated with intraocular inflammation; however, the mechanism is unknown and may itself be related to an improved immune status as suggested by Akler et al and Davis et al. ${ }^{27}{ }^{28}$ Three patients in our series were treated with intravenous cidofovir. In each of these patients the vitreous inflammatory reaction developed before starting cidofovir. In patients 1 and 2 the vitreous inflammatory reaction increased after starting intravenous cidofovir and CMO developed. In patient 1 during treatment with intravenous cidofovir there was persistent bilateral CMO. This was successfully controlled with oral acetazolamide. The CMO recurred when acetazolamide treatment was withdrawn and the patient remained on intravenous cidofovir, and resolved when acetazolamide was reintroduced. The CMO resolved permanently after discontinuing cidofovir. This is an association that has not previously been reported. In patient 6 intravenous cidofovir treated the active $\mathrm{CMV}$ retinitis and was associated with a reduction in the vitreous reaction. This may have been due to a reduction in the load of antigenic stimuli. A similar mechanism may have been responsible for improvement in patient 7 after treatment with intravitreal ganciclovir. The CMO and vitritis that was resistant to treatment with orbital floor steroids and acetazolamide improved despite no clinically evident active CMV retinitis.

This series suggests that orbital floor steroids are a useful and safe treatment in patients with a fall in visual acuity associated with IRV and that their use in this setting does not appear to be associated with a relapse of CMV retinitis. In patients with inactive CMV retinitis other causes of vitreous inflammation such as tuberculosis, syphilis, toxoplasmosis, lymphoma, and drug related inflammation must be excluded. Any active CMV retinitis should be fully treated with anti-CMV therapy. It must be stressed that patients developing such severe inflammation were a small minority of those with IRV and in most cases it probably remains a benign self limiting condition.

1 Karravellas MP, Lowder CY, Macdonald JC, et al. Immune covery vitritis associated with cytomegalovirus retinitis. Arch Ophthalmol 1998;116:169-75.

2 Zegans ME, Walton RC, Holland GN, et al. Transient vitreous inflammatory reactions associated with combination ntiretroviral therapy in patients with AIDS and cytomegalovirus retinitis. Am F Ophthalmol 1998;125:292-300.

3 Hoover DR, Peng Y, Saah A, et al. Occurrence of cytomegaovirus retinitis after human immunodeficiency virus immunosuppression. Arch Ophthalmol 1996;114:821-7.

4 Kupperman BD, Petty JG, Richman DD, et al. Correlation between $\mathrm{CD}^{+}$counts and prevalence of cytomegalovirus retinitis and human immunodeficiency virus-related noninfectious retinal vasculopathy in patients with acquired immus 575-82.

5 Rosberger DF, Heinemann MH, Freidgerg DN, et al. Uveitis associated with human immunodeficiency virus. Am $\mathcal{F}$ Ophthalmol 1998;25:301-5.

6 Silverstein BE, Smith JH, Sykes SO, et al. Cystoid macular edema associated with cytomegalovirus retinitis in patients with acquired immunodeficiency syndrome. Am f Ophthalmol 1998;25:411-15.

7 Palastine AG, Rodrigues MM, Macher AM, et al. Ophthalmic involvement in acquired immunodeficiency syndrome. Ophthalmology 1984;91:1092-9.

8 Stein D, Drusano G, Steigbigel R, et al. Two year follow-up of patients treated with indinavir $800 \mathrm{mg} \mathrm{q} 8 \mathrm{~h}$. Presented at the Fourth Conference on retroviruses and opportunistic infections. Washington DC, 1997;22-26 Jan:100 (abstract no 195).

9 Lederman M, Connici E, Landay A, et al. Partial immune reconstitution after 12 weeks of HAART (AZT, 3TC, (t) the Fourth Conference on retroviruses and opportunistic infections. Washington DC, 1997;22-26 Jan:208 (abstract
no LB13).

10 Jacobson MA, Zegans M, Pavan PR, et al. Cytomegalovirus retinitis after initiation of highly active antiretroviral therapy. Lancet 1997;349:1443-5. 
11 Holland GN, Pepose JS, Pettit TH, et al. Acquired immune deficiency syndrome. Ocular manifestations. Ophthalmoldeficiency syndrome.
ogy 1993;90:859-73.

12 Stenson SM, Friedberg DN. AIDS and the eye. New Orleans: Contact Lens Association of Ophthalmologists, 1995.

13 Jabs DA, Green WR, Fox R, et al. Ocular manifestations of acquired immune deficiency syndrome. Ophthalmology 1989;96:1092-9.

14 Kuo IC, Kapusta MA, Rao NA, Uveitis as the primary manifestation of ocular syphilis in patients with HIV inection. Am ₹ Ophthalmol 1998;125:306-11.

15 Nelson MR, Erskine D, Hawkins DA, et al. Treatment with corticosteroids: a risk factor for the development of clinical CMV disease in AIDS. AIDS 1993;7:375-8.

16 Bloch-Michel E, Nussenblatt RB. International uveitis study group recommendations for the evaluation of intra-ocular inflammatory disease. Am f Ophthalmol 1987;103:234.

17 Whitcup SM, Fortin E, Nussenblatt RB, et al. Therapeutic effect of combination antiretroviral therapy on cytomegaloeffect of combination antiretroviral therap
virus retinitis. $7 A M A$ 1997;277:1519-20.

18 Tural C, Romeu J, Sirera G, et al. Long-lasting remission of cytomegalovirus retinitis without maintenance therapy in human immunodeficiency virus-infected patients. F Infect

19 Reed JB, Schwab IR, Gordon J, et al. Regression of cytomegalovirus retinitis associated with protease-inhibitor treatment in patients with AIDS. Am $\mathcal{F}$ Ophthalmol 1997;124 199-205

20 Mitchell SM, Youle MS, Membrey WL, et al. Cytomegalovirus retinitis after initiation of highly active antiretroviral therapy. Lancet 1997;350:588.
21 Walsh JC, Jones CD, Barnes EA, et al. Increasing survival in AIDS patients with cytomegalovirus retinitis treated with combination antiretroviral therapy including HIV protease inhibitors. AIDS 1998;12:613-18.

22 Egbert PR, Pollard RB, Gallagher JG, et al. Cytomegalovirus retinitis in immunocompromised hosts. Ann Intern Med 1980; 93:664-70.

23 Connors M, Kovacs JA, Krevat S, et al. HIV infection induces changes in $\mathrm{CD} 4^{+} \mathrm{T}$-cell phenotype and depletions within the $\mathrm{CD} 4^{+} \mathrm{T}$-cell repertoire that are not immediately restored by antiviral or immune-based therapies. Nature restored by antiviral
Med $1997 ; 3: 533-40$

24 Godfrey WA, Smith RE, Kimura SJ. Chronic cyclitis: corticosteroid therapy. Trans Am Ophthalmol Soc 1977;74: 178-88.

25 Cox SN, Hay E, Bird AC. Treatment of chronic macular edema with acetazolamide. Arch Ophthalmol 1988;106: 1190-5.

26 Farber MD, Lam S, Tessler HH, et al. Reduction of macular oedema by acetazolamide in patients with chronic iridocyclitis: a randomised prospective crossover study. $\mathrm{BrF}$ Ophthalmol 1994;78:4-7.

27 Akler ME, Johnson DW, Burman WJ, et al. Anterior uveitis and hypotony after intravenous cidofovir for the treatment of cytomegalovirus retinitis. Ophthalmology 1998;105:6517.

28 Davis JL, Tashintuna I, Freeman WR, et al. Iritis and hypotony after treatment with intravenous cidofovir for cytomegalovirus retinitis. Arch Ophthalmol 1997;115: 733-7. 